\title{
Does complementary and alternative therapy work for SARS-CoV-2 (COVID-19)?
}

\author{
Sunil S Nikose ${ }^{1 *}$, Devashree Nikose ${ }^{2}$, Aditya Kekatpure ${ }^{1}$, Abhiram Awasthi ${ }^{1}$, Shashank Jain ${ }^{1}$ and Vrushabh Kumbhre ${ }^{1}$ \\ ${ }^{1}$ Department of Orthopedic Surgery and Director of Centre of simulation Studies: Jawaharlal Nehru Medical College, Sawangi - Meghe, Wardha, India \\ ${ }^{2}$ NKP Salve Institute of Medical Sciences, Nagpur, India
}

\begin{abstract}
Amidst the mid of December 2019, a novel coronavirus surfaced in the Wuhan region of China causing outbreaks of respiratory diseases along with assuming a pandemic proportion throughout the world due to its incredibly high airborne dissemination potential. Owing to the lack of efficient and specific treatments and the need to contain the epidemic", alternative therapies including herbal and Phyto medications, Chinese drugs, convalescent plasma therapy, and vitamin $\mathrm{C}$ and $\mathrm{D}$ were relied upon for the management of this SARS-CoV pandemic. Drug repurposing and repositioning strategies were also adopted. These alternative therapies may set the stage towards a supplemental alternative and all-inclusive treatment of COVID-19 till a safe, tested and long-lasting protection via a vaccine becomes available for public use.
\end{abstract}

\begin{abstract}
Abbreviations: SARS-CoV: Severe acute respiratory syndrome corona virus; COVID-19: Coronavirus disease 19; FDA: Food and drug administration; RNA: Ribonucleic acid; CP: Convalescent plasma; IU: International units; IV: Intravenous; CM: Chinese medicines/ medications; MAPK: Mitogen-activated protein kinase; PI3K: Class I phosphoinositide 3-kinase isoforms; mTOR: Mammalian Target of Rapamycin kinase/mechanistic Target of Rapamycin; SGK1: Serine/ threonine-protein kinase; IL- 6R: Interleukin 6 receptor also known as CD126 (Cluster of Differentiation 126); AKG: Alkylglycerols (AKGs); TGF- $\beta$ : Transforming growth factor-beta (TGF- $\beta$ ); EMT: Epithelial to mesenchymal transition; CQ: Chloroquine; HCQs: Hydroxychloroquines; MRSA: Methicillin-Resistant Staphylococcus aureus; Mm: Micrometer; mg: Milligrams; WHO: World health organization; SLAM: Signaling lymphocytic activation molecule; Vero: Verda Reno which means "green kidney" in Esperanto; pCXN2: Vero cells with a vector plasmid; pCAG-Hslam: Expression plasmid encoding the human signaling lymphocytic activation molecule.
\end{abstract}

\section{Introduction}

Amidst the mid of December 2019 a novel coronavirus surfaced in wet markets of Wuhan province of China causing flare-ups of acute and severe respiratory illness along with pandemic spread within a few weeks' time due to its high airborne transmission potential $[1,2]$. Healthcare staff and workers are under tremendous psychological pressure and are at higher risk of developing infections. Moreover, the nature of transmission during pregnancy has been a topic of another debate and vertical transmission of COVID - 19 is a likely possibility [3]. The virus was named as severe acute respiratory syndrome coronavirus -2 (SARS-CoV-2) (formerly 2019 - CoV) and is liable for respiratory contaminations including acute pneumonia along with a variable death rate across nations in the range of around $2.6-3.5 \%$ which has a tendency to increase in the elderly population and those people with co-morbid conditions. [4]. SARS-CoV-2 is a "high mutant virus" and human to human transmission is unprecedented and rapid alongside these zoonotic pathogens influencing numerous animal species. The clinical highlights of Coronavirus disease extend from being asymptomatic to the need for hospitalizations in emergency units requiring intubation and ventilation for severe forms of respiratory disease and the infection predominantly influences respiratory, hepatic, neurological, cardiac, and gastrointestinal systems. Till date our knowledge concentrates on the four kinds of coronaviruses namely - NL63 (alpha) belongs to group 1 Coronavirus, HKU1 (beta) and OC43 (beta) belongs to Group 2a bovine coronavirus and 229E (alpha) which belongs to group 1 coronavirus are usually in circulation in humans and usually cause mild respiratory diseases $[5,6]$. The restricted time period permitted to confront this COVID-19 pandemic besides its seriousness demands and indefatigable commitment from the established researchers to rediscover and reframe alternative therapeutic approaches by way of medications and nutraceuticals, vitamins, herbal formulas, novel medications, and sequenced medications which might be helpful during this time in any way towards curbing this pandemic and reducing morbidity and mortality. This novel coronavirus has been in conflict regarding its origin in published and unpublished works and it is felt that despite a fast- track progress regarding the vaccine by various countries it is expected to last for a longer period of time [7]. Besides, COVID-19 is pre sently still far from control and without a known approved effective therapy efforts of the laboratories and medical teams across continents have concentrated on repurposing the strategy guided by the United States Food and Drug Administration (FDA) endorsed

${ }^{\star}$ Correspondence to: Sunil S Nikose, Professor, Department of Orthopedic Surgery and Director of Centre of simulation Studies: Jawaharlal Nehru Medical College, Sawangi - Meghe, Wardha, India, Tel: +919881555017; E-mail: sunilnikose@gmail.com

Key words: SARS-CoV pandemic, alternative drug therapy, convalescent plasma therapy, vitamin $c$, herbal medicines

Received: September 02, 2020; Accepted: September 18, 2020; Published: September 21, 2020 
medications to treat COVID-19 cases by various permutations and combinations with no certain guidelines across the globe which are being followed due to an inconsistent approach which is stuck between health and economic policies and thereby the medication which are helpful in repurposing a disease is thought to be an effective method to rapidly identify therapeutic drugs with a recognized protective outline to aim to treat sickness due to COVID-19 [8].

Likewise, with several other communicable and spreading pandemic indisposition, immunization commitment is thought to be capable of generating a secure and shielded enduring immunity from SARS-CoV-2 disease, however, the approach seems inappropriate during the current COVID-19 flare-up. As an alternative approach a natural Chinese medication is promoted which has a theoretical potential to modulate the host resistance and immune response. Nonetheless, the amalgamation and complete clinical investigations from preclinical studies to phase III clinical trials of the new therapeutics is a challenging task and laborious during the present international catastrophe [9]. Hence, repurposing and or repositioning of drugs represents a reasonable substitute, presuming that suitable medications are designated amongst the various medications previously incorporated, readily available in the market, and often formerly therapeutically used medications for a variety of illnesses with a known safety threshold. Medication repurposing and remodelling methods have been earlier proposed since the outbreak of pandemic for specific and non- specific medications in the management of the present-day COVID-19 episodes [10,11]. A large number of medications which are judiciously investigated towards repurposing and or repositioning strategy for management of the COVID-19 flare-ups are economically accessible with the prescription measurements along with the minimum and maximum dosage schedule and toxic effects of the drugs are appropriately researched and understood which are attributable to their clinical use over the years. This would permit its usage within the quicker window of time in addition to a more affordable and practical stage II or III therapeutic clinical preliminaries together with a straight forward beneficent application.

\section{The natural therapeutic approach towards COVID-19}

The utilization of natural herbal products and phytomedicine were powerful in lessening infectious conditions and were the foremost medications available before antibiotics were discovered. Specifically, herbal medicinal commodities provide sustenance mechanisms for the manufacturing of innovative medications curative for viral diseases. Cheng at al have reported normally occurring triterpene glycosides (saikosaponins A, B, C, and D) isolated from herbal plants, such as Heteromorpha spp., Bupleurumspp, and Scrophulariascorodonia, have antiviral properties against $\mathrm{HCoV}-22 \mathrm{E} 9$ [12]. These herbal products were discovered to be effectively preventing the beginning phase of HCoV-22E9 disease by affecting viral penetration and attachment. Concentrates from Artemisia annua, Lycoris emanate, Pyrrosia lingua and Linderatotal have likewise been accounted for the exposition of anti-SARS-CoV impacts against a backdrop of screening programs and investigations utilizing a large number of Chinese therapeutic spices [13]. The watery concentrate from Houttuyniacordata, which has been particularly found to show diverse antiviral systems against SARS-CoV that incorporate hindering the viral $3 \mathrm{CL}$ protease and obstructing the movement of viral RNA- dependent RNA polymerase as demonstrated in Figure 1 [14].

\section{The role of convalescent plasma in COVID-19 infection}

This empirical and theoretical achievement soundness regarding plasma therapy management is assigned by the means of making use of immune responses from the recuperated COVID-19 patient's blood to manage these critically ill patients affected by the virus. The abovementioned treatment has therefore been employed across various nations, especially in developing nations like India as a safeguard for those individuals who are at higher risk for development of severe respiratory disease. The recommended target donors of plasma should be otherwise healthy people with good protein intake, relatives of patients, and non-identical contacts who are thought to be at high risk of exposure to the coronavirus infection in the immediate past

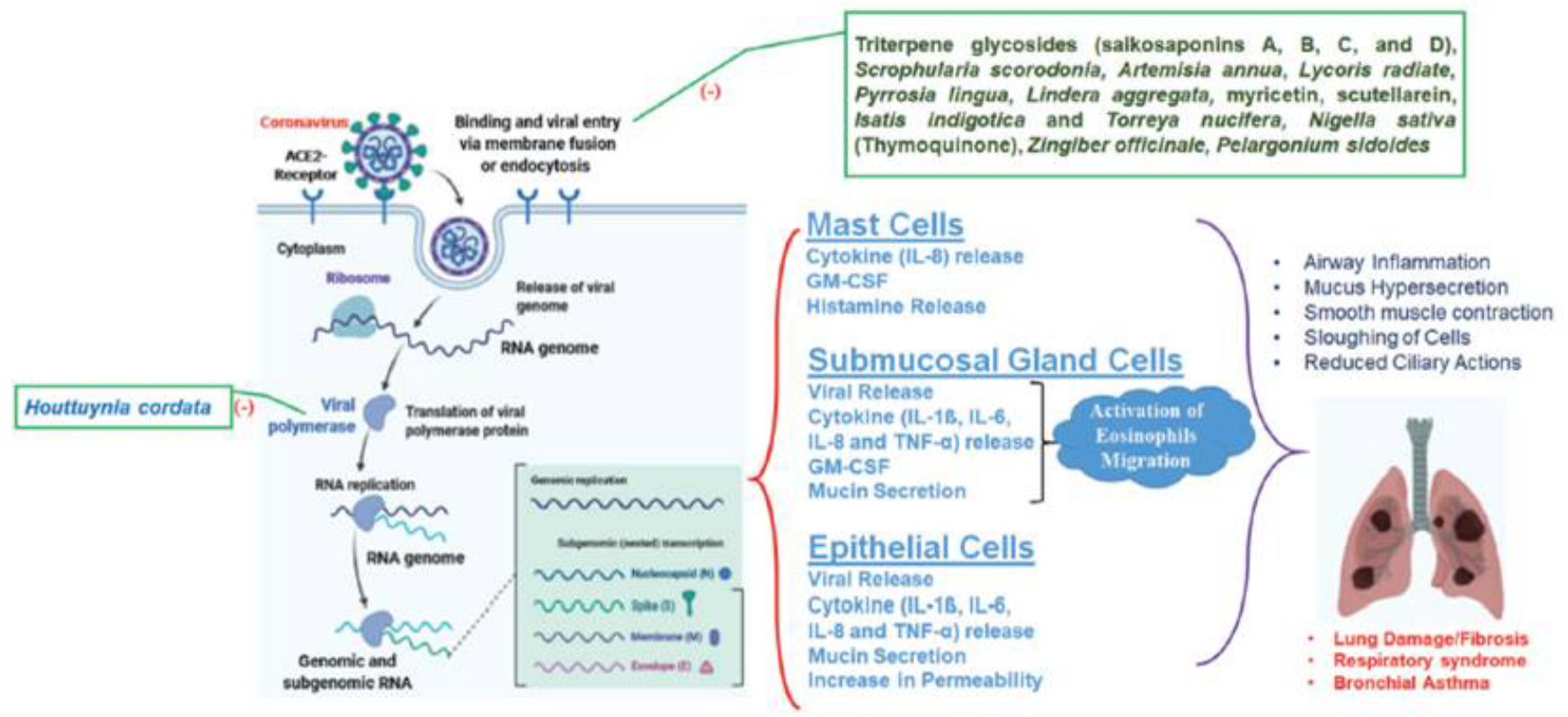

Figure 1. Mechanism of action of Houttuyniacordatain COVID-19 infection 
period of a month or so. This treatment's basic idea is established on the basis of the subsistence assuming the blood of a patient who has recouped from COVID-19 presumably comprises of antibodies with the particular capacity of battling novel coronavirus. The convalescent plasma treatment is much the same as numerous other vaccinations as dictated by the specialists' analogy that it is a preventive measure and not a treatment for the COVID-19 illness [15]. A single portion of approximately $200 \mathrm{~mL}$ of Convalescent Plasma (CP) is obtained from an individual who has been recuperated from COVID -19 along with a neutralizing antibody (Anti-SARS-CoV-2) titres above 1:640 were transfused to the patients as an additive to supportive care along with the antiviral drugs [16]. It is believed that this seroconversion of titres is not universal and is usually observed after a median of 10-13 days after the symptom onset for IgM and around 12 to 14 days for the IgG. However, how long this lasts is a matter of question as different individuals have demonstrated different levels of Anti-SARS-CoV-2 antibodies and are highly variable with negative tests not ruling out the possibility of infection with SARS-CoV -2 . The essential target, as advocated by researchers, is the harmlessness of CP transfusion with a subsequent boost of clinical improvements and improved laboratory values within the 72 hours window following a CP countermeasure therapy. The CP derived from recouped COVID-19 individuals who had inset up humoral susceptibility against the infection contains a huge amount of lethal viral antibodies for the body's defense equipped with killing capacity for SARS-CoV-2 resulting in destroying the virus from blood and pulmonary tissues [17]. However, not all countries would advocate this measure as a preventive or therapeutic measure given the inadequate evidence for this tool.

\section{Therapeutic role of vitamins in COVID-19}

There is ample evidence to suggest that vitamin D might help to protect against getting contaminated by developing serious symptoms of COVID-19. Vitamin D may ensure protection against COVID-19 by two different ways such as to start with it might help boost our bodies' natural defenses against viruses and bacteria and secondly, it has a capability to help prevent an overwhelming inflammatory counterattack which has been instrumental in defense against severe illness in few COVID-19 patients [18].

The suggested dietary dosage of vitamin D is 600 International Units (IU) every day for grown-ups less than 70 years old and $800 \mathrm{IU}$ every day for the elderly population who are more than 70 years. A daily supplement containing 1000 to 2,000 IU of vitamin D is likely safe for the majority of patients. Whereas, one of the greatest significant exemplifications of life-threatening SARS-CoV-2 contamination is lymphopenia and in this condition the mice models and human cell lines demonstrated defensive effects on the interstitial pneumonitis after administration of Vitamin D [19,20].

A meta-analysis in early 2020 have shown that intravenous (IV) high-dose ascorbic acid (vitamin C) treatment has considerable advantages during the management of critical COVID-19 related to Adult Respiratory Distress Syndrome (ARDS) and it was concluded that the patients who were suffering from COVID-19 and hospitalized with breathing trouble, impaired biomarkers, and impending sepsis appeared to be ideally suited candidates for a short term intravenous vitamin $C$ therapy administered in high doses during the initial phase of the disease [21]. High-dose IV ascorbic acid dosage is typically administered at a four to six hourly dosages of $50 \mathrm{mg} /$ per kilogram body weight for around four days along with a restriction of glucose intake it becomes a more potent antioxidant and cofactor for the biosynthesis of essential enzymes acting as a phagocytic molecule and gene regulatory mechanism. In the parenteral form and in high dosages in COVID-19 infection it acts to prevent oxidative stress, acts pleiotropically as a favourable pro-oxidant, enhances chemotaxis, decreases iron-inflammatory mediator expression improves alveolar liquid clearance, and acts as an immune modulator while improving the functions of epithelial cells [22].

\section{Role of Chinese medicines in the prevention and treatment of SARS-CoV infections}

After the COVID-19 outbreak happened several preventive programs suggested the utilization of Chinese medication (CM) and subsequently, their use is on the rise around the world. The primary fundamental concept in the use of CM in forestalling COVID-19 was to identify vital energy to shield and give protection from outside microbes, scatter wind, release warmth, and resolve dampness with fragrance [23]. The six most informally utilized spices were Astragali Radix (Huangqi), Glycyrrhizae Radix Et Rhizoma (Gancao), Saposhnikoviae Radix (Fangfeng), Atractylodis Macrocephalae Rhizoma (Baizhu), Lonicerae Japonicae Flos (Jinyinhua) and ForsythiaeFructus (Lianqiao). The Astragali Radix (Huangqi), Saposhnikoviae Radix (Fangfeng), and Atractylodis Macrocephalae Rhizoma (Baizhu) are the constituents of an old pure balanced natural formula of Yupingfeng mixture powder for tonifying vitality while shielding against the virulent foreign microorganisms [24]. Numerous investigations and research have documented that Yupingfeng powder has antiviral, anti-inflammatory, and immunoregulatory impacts $[25,26]$. JaponicaeFlos (Jinyinhua) and ForsythiaeFructus (Lianqiao) are the core parts of Yinqiao Powder, which was a classical formula used to prevent and manage common cold, flu, and even moderate respiratory illness during ancient times. However, during the current pandemic, the National Health Commission of China has neither permitted nor allowed the representation of CMs in the prevention program or management of COVID-19 infection. The reasons might be many and the justified reason pointed out is that the Chinese Medicines usually favours and support three inherent factors based on individual, regional, and seasonal factors and these differences throughout the world have vast variances in these three factors and subsequently, it would not be suitable for either prevention or treatment of COVID-19 disease [27].

\section{Repurposing/repositioning drug strategy for SARS- CoV infection}

Conceivably suitable medications that are en route to repositioning are basically the ones that influence either signal transduction, a combination of macromolecules and add bioenergetics pathways which can then have the ability to impede the immune reaction of the host, accompanied by a severe life-threatening cytokine storm related to extreme COVID-19 infection and lastly medications which have known to be chemotherapeutic in malignancy as depicted in Table 1.

\section{Chloroquine (CQ) and hydroxychloroquine (HCQs)}

CQ and HCQs have demonstrated anti-infective properties against varying organisms and act as a broad-spectrum antibiotic along with possession of variable anti-viral properties, particularly concerning flaviviruses, retroviruses, and coronaviruses [28,29]. The HCQ molecule interferes with biosynthesis of sialic acid while disconcerting the adjustments regarding post-translational transmembrane of the viral restricting proteins in such a way that it hinders the viral entrance within the cells thus demonstrating the therapeutic effect against the 
Table 1. Alternative drugs tested for SARS-CoV infection

\begin{tabular}{|l|l|}
\hline Drug mechanism of action & \\
\hline Rapamycin and its derivatives & Immunosuppressant; P13K/mTOR inhibitor; viral replication inhibitors \\
\hline Chloroquine and its derivatives & $\begin{array}{l}\text { Anti-infective with broader coverage; antimalarial; interfering with the process of protein post-translational; Inhibitor of autophagy; inhibiting MAPK; } \\
\text { pro-inflammatory cytokines inhibitor. }\end{array}$ \\
\hline S113 & SGK1 inhibitor \\
\hline Tocilizumab & Monoclonal antibodies which target and sequence IL-6R, thereby preventing cytokine storms and degeneration of fibrin products. \\
\hline Sarilumab & Monoclonal antibodies targets IL-6R, thus preventing cytokine storms along with fibrotic degeneration. \\
\hline Anakinra plus Empalumab & Monoclonal antibodies targeting AKG2A \\
\hline Bacillus Calmette Guerin & Prevention of tuberculosis; inhibits a TGF- $\beta 1$ mediated EMT \\
\hline Ritonavir plus Lopiravir & Inhibitor of Viral Protease and recommended for HIV treatment \\
\hline Ribavirin & Affects synthesis of RNA of the virus, Inhibiting RNA dependent RNA polymerase chain \\
\hline Remdesivir & Inhibits Viral RNA polymerase chain \\
\hline
\end{tabular}

MAPK: Mitogen-activated protein kinase; PI3K: Class I phosphoinositide 3-kinase isoforms; Mtor: Mammalian target of rapamycin kinase; SGK1: Serine/threonine-protein kinase; IL6R: Interleukin 6 receptor also known as CD126 (Cluster of Differentiation 126); AKG: Aalkylglycerols (AKGs); TGF- $\beta$ : Transforming growth factor-beta (TGF- $\beta$ ); EMT: Epithelial to mesenchymal transition; RNA: Ribonucleic acid.

viruses. The association between SARS-CoV-2 and the film receptor angiotensin-converting enzyme II (ACE II) is primarily present within the alveolar epithelial cells of the lung, within the enterocytes of the small intestine, Leydig cells and Sertoli cells relying strongly on glycosylation [30]. HCQ likewise contributes to the alkalization of endothelial cells and thereby enhances the degradation of the viral particles due to the enzymatic process of degradation by proteases in addition to slowing down the process of endocytosis a fundamental which is absolutely necessary for the utilizable nucleic acid of the virus. Moreover, HCQ improves the presentation of viral immune trigger and thereby helps in the up-gradation of T-cell-mediated immunity in addition to its competency in terms of regulating the provocative erythrogenic stimulation consequently scaling down the amalgamation of cytokines which are favorable for inflammation. However, after the exponential use of HCQs by various nations in the preventive measures and prophylaxis, it fell into disrepute due to cardiovascular adverse events. The HCQ dramatically reduces the antiviral effect of the drug Remedesivir which must be kept in mind by healthcare professionals who prescribe the medications and the combination of these two drugs have been coined as dangerous by the FDA. The initial use of HCQ along with azithromycin was reported to have posed adverse threats to the cardiovascular events by pathological prolongation of QT interval on electrocardiogram (ECG) due to synergistic effects on QT interval resulting in lethal arrhythmias and subsequently it was reported by the FDA that there is an associated increase of mortality due to cardiovascular causes, increase in incidences of angina chest pain and increased heart failure. The WHO arm of solidarity trial concerning the HCQs in COVID-19 was stopped abruptly on 17 June 2020. However, The Indian Council of Medical Research (ICMR) continues to advocate the use of HCQs for asymptomatic healthcare professionals, their family members and contacts and for confirmed cases of COVID-19 who develop mild to moderate symptoms along with monitoring of ECG in high-risk individuals likely to develop adverse cardiovascular events.

\section{Tocilizumab}

The compound was introduced as an inhibitor of Interleukin - 6 (IL6) and was a part of the protocol for investigation against COVID-19 as a humanized monoclonal antibody targeting interleukin- 6 receptor (IL-6R). The initial reports of Phase III clinical trials were encouraging, which revealed that it is safe and effective in hospitalized patients and demonstrates its efficacy by limiting the cytokine storm derived from SARS-CoV-2 infection. This medication is at present subject to assessment at various therapeutic levels on its operational acceptability in addition to offering protection of severity in patients with COVID-19 pneumonia [31]. Although the trials did not meet the primary endpoint improvement of the clinical state of COVID-19 pneumonia patients or the secondary endpoint in reducing the deaths, it demonstrated a positive correlation of early hospital discharge in patients who received this drug. Therefore the FDA (USA) has established that it can be tried safely and already green signal has been provided for its controlled investigation so that the efficacy of tocilizumab can be established in critical patients within the confines of the Intensive care unit (ICU) patients presenting with fulminating pneumonia.

\section{Rapamycin}

As regards rapamycin after initially successfully testing for antifungal properties it also demonstrated a very good immunosuppressive effect in organ-transplanted individuals and in the past few months it has been established as a cytotoxic agent Rapamycin alone or in combinations with Actinomycin -D had been recently proposed to interfere with the SARS-CoV-2 interactome in a systems pharmacology-based network medicine platform [32]. Similarly, due to its immunosuppressant effects, it theoretically has an appropriate relevance in alleviating inflammatory mediation due to SARS-CoV-2. The Serine/threonine kinase mTOR (mechanistic Target of Rapamycin) in SARS-CoV-2 has regulatory properties for reducing hyper inflammation, cytokine storm within the host immune system by entering the target organ (lungs) via droplets thereby blocking mTOR and limiting IL- $\beta$, restricting the production of inflammatory mediators IL-6 including pyroptosis.

\section{Teicoplanin}

Teicoplanin is a powerful antibiotic, mainly has an action against the resistant Gram-positive organisms including the management of hospitalized patients with Methicillin-Resistant Staphylococcus aureus (MRSA) and is a semisynthetic derivative of glycopeptide which was found to be possessing complementary antiviral properties and its efficacy against SARS -CoV-2 in vitro maintains its position as an inventory molecule that could be employed as a therapeutic array competing with COVID-19. According to Zhou et al. Teicoplanin functions as an antiviral at the starting phase of the life cycle of coronavirus by repressing the low-pH separation of the spike of coronavirus protein by cathepsin $\mathrm{L}$ in the late endosomes thereby preventing the viral multiplication cycle due to intercepting the release of the genomic viral RNA. The Minimum Inhibitory Concentration (MIC 50) of Teicoplanin in vitro is $1.66 \mu \mathrm{M}$ which is considerably reduced when it has to be administered intravenously in the human blood, which is $8.78 \mu \mathrm{M}$ for a day by day portion of $400 \mathrm{mg}[33,34]$. 


\section{Ivermectin in addition to Doxycycline}

Ivermectin is an FDA approved antiparasitic medication and is in the World Health Organization (WHO) list of essential medications and pharmacologically it is a macrocyclic lactone which demonstrates a wide range of antiviral activity [35]. It is a reasonably safe drug which possesses the highest potency along with the class of avermectins and is a semi-synthetic derivative. Ivermectin likewise being an incredible antiviral and therefore it has been additionally recommended for the potential treatment of the new SARS-CoV-2 related condition with a reported 5,000- times overlap decrease in SARS-CoV-2 RNA viral load levels in comparison to those patients who were in the control group after being infected Vero/hSLAM cells (Vero/hSLAM are derived from transfection of Vero cells with a vector plasmid (pCXN2) incorporating the gene responsible for neomycin resistance along with the plasmid expression (pCAG-hSLAM) which has the property to encode the signalling lymphocytic activation molecule (SLAM) in humans, also known as CDw150 and which acquired viral infections for 48 hours with $5 \mu \mathrm{M}$ of Ivermectin [36]. With respect to its character as an antiviral drug, it possesses the requisite viability of a few infections both in vitro and in vivo. There are numerous mechanisms postulated as far as the role of this drug is concerned but the paramount importance has been advocated as its role in the nuclear transport mechanism which is intervened by the importin $\alpha / \beta 1$ heterodimer and dependable as regards the movement of different proteins component of coronavirus and various other viruses (HIV-1, SV40) which are very vital for their replication $[37,38]$. Due to this property of inhibitory mechanism, it influences a substantial number of RNA viral infections since the contributory organism for COVID-19 is also happens to be an RNA virus infection, and it is well expected that Ivermectin will also exhibit its activity alongside the aforementioned proteins, polypeptide chains, and molecular processes [39].

The synergistic effect of Ivermectin (anti-parasitic) and doxycycline (anti-toxin) is a superior combination of COVID diseases. Doxycycline, in addition to its antibiotic properties exhibits anti-inflammatory and antiviral effects in vitro against a few RNA infections. The utilization of doxycycline has been related to clinical improvement including inversion of cytokine storm in certain diseases brought about by RNA infections, for example, SARS-CoV infection [40]. How the Doxycycline molecule demonstrates it anti-viral properties is a matter of theoretical concern but it works as an auxiliary molecule for causing transcriptional upregulation within the intracellular zinc-finger antiviral protein (ZAP) which is an encoding quality within the cells of the host [41]. ZAP presumably is capable of binding to a particular target in viral mRNAs along with the suppression and transplanting rephrasing of RNAs.

\section{Conclusion}

The COVID-19 pandemic period and the present day has found the world certainly ill-equipped to deal with such a worldwide crisis. The forcefully limited number of complementary and alternative elective medication treatments quickly portrayed in this review indicates some exceptionally promising outcomes and seems to assist the world in battling the SARS-CoV-2 infection until we get a lasting, safe protected and enduring immunization for the same. Although, the complementary and alternative therapies described above have a wide potential of safety requiring very little medical supervision for drug administration and can even be prescribed via telemedicine after evaluating the less severely ill patients that would beneficially contribute immensely to human health and welfare during these unprecedented times of COVID-19. However, local, regional and national guidelines are to be followed for the safety of the individuals.

\section{Acknowledgements}

All the authors would like to express their great sense of appreciation to Miss Bhagyashree Nikose for her technical support during the preparation of this review article during the peak phase of COVID - 19 pandemic. She was instrumental in collection of internet and web data, from PubMed, research gate, google, ICMR and multiple search engine. This was her voluntary "advanced internship summer project" regarding orientation of medical sciences due to extended lockdown and closure of schools in the Indian Subcontinent.

\section{Authors' contributions}

SN and DN conceptualized the review; AA and SJ were involved in the literature search and study selection; AK and VK were involved in disagreement resolution and finalization of the included studies; $\mathrm{SN}$ and DN extracted data from the studies for qualitative synthesis of the evidence; $\mathrm{SN}, \mathrm{DN}, \mathrm{AK}$, and SJ interpreted the analyses; $\mathrm{SN}, \mathrm{DN}$, and $\mathrm{AK}$ drafted the review; $\mathrm{SN}$ revised the manuscript; DN provided with the expert input and updated the final review.

\section{Declarations}

Funding: None.

Conflicts of interest/Competing interests: The authors declare that they have no conflict of interest.

Availability of data and material: Not applicable.

Code availability: Not applicable.

Ethical approval: Not applicable.

Consent to participate: Not applicable.

Consent for publication: Not applicable.

\section{References}

1. Zhu N, Zhang D, Wang W, Li X, Yang B, et al. (2019) A novel coronavirus from patients with pneumonia in China, 2019. N Engl J Med 382: 727-733.

2. Lai CC, Shih TP, Ko WC, Tang HJ, Hsueh PR (2020) Severe acute respiratory syndrome, coronavirus 2 (SARS-CoV-2), and coronavirus disease-2019 (COVID-19): the epidemic and the challenges. Int J Antimicrob Agents 2020: 105924.

3. Sunil SN, Devashree N (2020) Vertical Transmission of COVID 19 during Pregnancy Glob J Reprod Med 8: 5556727.

4. Baron SA, Devaux C, Colson P, Raoult D, Rolain JM (2020) Teicoplanin: an alternative drug for the treatment of COVID-19? Int J Antimicrob Agents 55: 105944.

5. Salehi S, Abedi A, Balakrishnan S, Gholamrezanezhad A (2020) Coronavirus disease 2019 (COVID-19): A systematic review of imaging findings in 919 patients. Am J Roentgenol.

6. Singhal T (2020) A review of coronavirus disease-2019 (COVID-19). Indian J Pediatr 87: 281-286.

7. Sunil SN, Devashree N, Shashank J, Kiran S, Aditya K, et al. (2020) COVID -19 and Orthopedic Surgery: Strategies from Freeze to the Light at the End of the Tunnel. Glob J Ortho Res 2: 2020

8. Ciliberto G, Rita Mancini R, Paggi MG (2020) Drug repurposing against COVID-19: Focus on anticancer agents. Journal of Experimental \& Clinical Cancer Research 39: 86

9. Elfiky AA (2020) Anti-HCV, nucleotide inhibitors, repurposing against COVID-19 Life Sci 248: 117477.

10. Zhou Y, Hou Y, Shen J, Huang Y, Martin W, et al. (2020) Network-based drug repurposing for novel coronavirus 2019-nCoV/SARS-CoV-2. Cell Discov 6: 14. 
11. Fan HH, Wang LQ, Liu WL, An XP, Liu ZD, et al. (2020) Repurposing of clinically approved drugs for treatment of coronavirus disease 2019 in a 2019-novel coronavirus (2019-nCoV) related coronavirus model. Chin Med J.

12. Cheng PW, Ng LT, Chiang LC, Lin CC (2006) Antiviral effects of saikosaponins on human coronavirus 229E in vitro. Clin Exp Pharmacol Physiol 33: 612-616.

13. Li SY, Chen C, Zhang HQ, Guo HY, Wang H, et al. (2005) Identification of natural compounds with antiviral activities against SARS-associated coronavirus. Antiviral Res 67: 18-23

14. Lau KM, Lee KM, Koon CM, Cheung CS, Lau CP, et al. (2008) Immunomodulatory and anti-SARS activities of Houttuyniacordata. J Ethnopharmacol 118: 79-85.

15. Duan K, Liu B, Li C, Zhang H, Yu T, et al. (2020) Effectiveness of convalescent plasma therapy in severe COVID-19 patients. Proc Natl AcadSci USA 117: 9490-9496.

16. Chen N, Zhou M, Dong X, Qu J, Gong F, et al. (2020) Epidemiological and clinical characteristics of 99 cases of 2019 novel coronavirus pneumonia in Wuhan, China: A descriptive study. Lancet 395: 507-513.

17. Marano G, Vaglio S, Pupella S, Facco G, Catalano L, et al. (2016) Convalescent plasma: New evidence for an old therapeutic tool? Blood Transfus 14: 152-157.

18. Ali N (2020) Role of vitamin D in preventing of COVID-19 infection, progression and severity. J Infect Public Health.

19. Tian Y, Rong L (2020) Covid-19 and vitamin D-authors' reply. Aliment Pharmacol Ther.

20. Holick MF (2017) The vitamin D deficiency pandemic: approaches for diagnosis, treatment and prevention. Rev Endocrine Metab Disord 18: 153-165.

21. Kashiouris MG, L'Heureux M, Cable CA, Fisher BJ, Leichtle SW, et al. (2020) The emerging role of vitamin $\mathrm{C}$ as a treatment for sepsis. Nutrients 22: E292.

22. Erol A (2020) High dose intravenous Vitamin C for COVID-19.

23. Luo H, Tang QL, Shang YX, Yang M, Robinson N, et al. (2020) Can Chinese medicine be used for prevention of corona virus disease 2019 (COVID-19)? A review of historical classics, research evidence and current prevention programs. Chin J Integr Med 26: 243-250. [Crossref]

24. Lau J, Leung P, Wong E, Fong C, Cheng K, et al. (2005) The use of an herbal formula by hospital care workers during the severe acute respiratory syndrome epidemic in Hong Kong to prevent severe acute respiratory. J Alternat Complement Med 11: 49-55.

25. Du C, Zheng K, Bi C, Dong T, Lin H, et al. (2015) San, an ancient Chinese herbal decoction, induces gene expression of anti-viral proteins and inhibits neuraminidase activity. Phytother Res 29: 656-661.

26. Gao J, Li J, Shao X, Jin Y, Lü X, et al. (2009) Anti-inflammatory and immunoregulatory effects of total glucosides of Yupingfeng Powder. Chin Med J 122: 1636-1641.
27. Ou AH, Lu CJ, Li JQ, Li XY, Wen ZH, et al. (2014) Analysis on the Chinese medicine syndromes and demographic characteristics of patients with influenza-like illness in clinics of China. Chin J Integr Med 20: 101-106.

28. Rolain JM, Colson P, Raoult D (2007) Recycling of chloroquine and its hydroxyl analogue to face bacterial, fungal and viral infections in the 21 st century. Int $J$ Antimicrob Agents 30: 297-308.

29. Savarino A, Boelaert JR, Cassone A, Majori G, Cauda R (2003) Effects of chloroquine on viral infections: an old drug against today's diseases? Lancet Infect Dis 3: 722-727.

30. Walls AC, Park YJ, Tortorici MA, Wall A, McGuire AT, et al. (2020) Structure, function, and antigenicity of the SARS-CoV-2 spike glycoprotein. Cell.

31. Xu X, Han M, Li T, Sun W, Wang D, et al. (2020) Effective treatment of severe COVID-19 patients with tocilizumab. Proc Natl AcadSci USA.

32. Zhou Y, Hou Y, Shen J, Huang Y, Martin W, et al. (2020) Network-based drug repurposing for novel coronavirus 2019-nCoV/SARS-CoV-2. Cell Discov 6: 14.

33. Baron SA, Devaux C, Colson P, Raoult D, Rolain JM (2020) Teicoplanin: an alternative drug for the treatment of COVID-19? International Journal of Antimicrobial Agents 55: 105944.

34. Zhou N, Pan T, Zhang J, Li Q, Zhang X, et al. (2016) Glycopeptide antibiotics potently inhibit cathepsin L in the late endosome/lysosome and block the entry of Ebola virus, Middle East respiratory syndrome coronavirus (MERS-CoV), and severe acute respiratory syndrome coronavirus (SARS-CoV). J Biol Chem 291: 9218-9232.

35. Gonzalez Canga A (2008) The pharmacokinetics and interactions of ivermectin in humans-a mini-review. AAPS J 10: 42-46.

36. Caly L, Druce J, Catton M, Jans D, KM W (2020) The FDA-approved Drug Ivermectin inhibits the replication of SARS-CoV-2 in vitro. Antiviral Res.

37. Wagstaff KM, Rawlinson SM, Hearps AC, Jans DA (2011) An AlphaScreen(R)-based assay for high-throughput screening for specific inhibitors of nuclear import. Journal of Biomolecular Screening 16: 192-200.

38. Wagstaff KM, Sivakumaran H, Heaton SM, Harrich D, Jans DA (2012) Ivermectin is a specific inhibitor of importin alpha/beta mediated nuclear import able to inhibit replication of HIV-1 and dengue virus. The Biochemical Journal 443: 851-856.

39. Jans DA, Martin AJ, Wagstaff KM (2019) Inhibitors of nuclear transport. Curr Opin Cell Biol 58: 50-60.

40. Castro JZ, Fredeking T (2010) Doxycycline modify the cytokine storm in patients with dengue and dengue hemorrhagic fever. Int J Infect Dis.

41. Tang Q, Wang X, Gao G (2017) The short form of the zinc finger antiviral protein inhibits Influenza - A virus protein expression and is antagonized by the virus-encoded NS1. J Virol.

Copyright: (C2020 Nikose SS. This is an open-access article distributed under the terms of the Creative Commons Attribution License, which permits unrestricted use, distribution, and reproduction in any medium, provided the original author and source are credited. 